\title{
HARMONIC COMPONENT DETECTION: OPTIMIZED SPECTRAL KURTOSIS FOR OPERATIONAL MODAL ANALYSIS
}

\author{
J.-L. DION, I. TAWFIQ, G.CHEVALLIER \\ Laboratoire d'Ingénierie des Systèmes Mécaniques et des Matériaux (LISMMA) \\ Institut supérieur de Mécanique de Paris 3, rue Fernand Hainaut \\ 93407 Saint Ouen France \\ jean-luc.dion@supmeca.fr tel: 33(0)1 49452912 fax : 33(0)1 49452910
}

\begin{abstract}
:
This work is a contribution in the field of Operational Modal Analysis to identify the modal parameters of mechanical structures using only measured responses. The study deals with structural responses coupled with harmonic components amplitude and frequency modulated in a short range, a common combination for mechanical systems with engines and other rotating machines in operation. These harmonic components generate misleading data interpreted erroneously by the classical methods used in OMA. The present work attempts to differentiate maxima in spectra stemming from harmonic components and structural modes. The detection method proposed is based on the so-called Optimized Spectral Kurtosis and compared with others definitions of Spectral Kurtosis described in the literature. After a parametric study of the method, a critical study is performed on numerical simulations and then on an experimental structure in operation in order to assess the method's performance.
\end{abstract}

Keywords: operational modal analysis, kurtosis, spectral kurtosis, detection 


\section{Acronyms:}

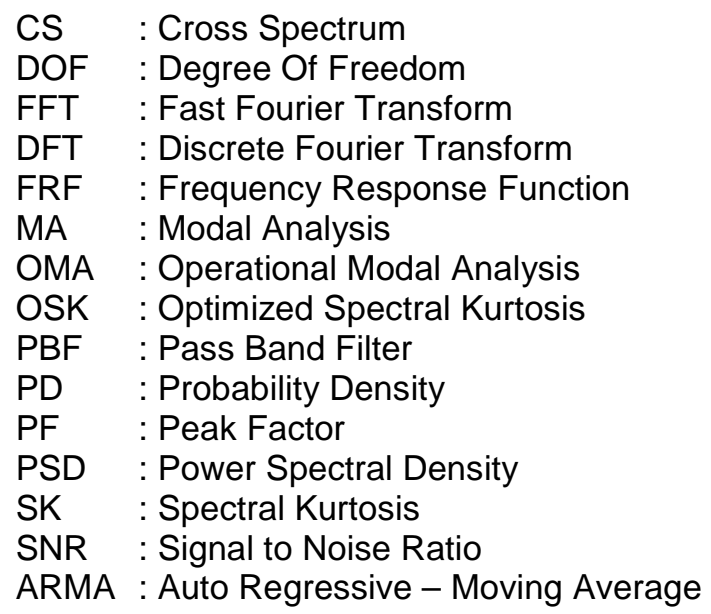

\section{Introduction}

Structural dynamics are used to determine the stability, safety and lifetime of mechanisms under normal operational conditions and to a great extent rely on modal analysis. Traditional modal analysis is a very well known tool used for identifying modal parameters that consists in applying a forced excitation on the structure being tested. By measuring the input and response signals, we can identify the modal parameters (resonance frequencies, damping ratio and mode shapes) of the structure, Ewins [1], Maia [2]. Modal parameters can also be obtained from classical Modal Analysis (MA) techniques by using the Frequency Response Function (FRF) and most dynamics problems can be solved efficiently by this method, Ewins [1]. In order to identify the modal parameters involved, this technique entails measuring the excitation forces and dynamic responses of the structure.

However, in certain cases, it is not possible to measure the excitation force, for example, during flight tests of aircraft and helicopters, and vibrations of buildings, bridges and offshore platforms.

For these reasons, Operational Modal Analysis (OMA) has been developed under the following assumption: only structural responses are known, whereas excitation forces are unknown.

Hermans [3] and Pintelon [4], assume that excitations are random. Another method, usually performed to solve industrial problems, identifies peak Power Spectral Densities (PSD) and Cross-Spectrums (CS) under operating conditions [5]. These peaks are then used to estimate natural frequencies and normal modes. However this technique does not allow identifying damping with good accuracy.

The "Natural excitation" technique (NEXT) [6] considers the excitation force as a stationary white noise.

Under this assumption, the Spectra and Cross Spectrum correspond to an impulse response. Modal parameters are determined from temporal responses with a complex exponential function [7], or from the Ibrahim method (ITD) [8] or from the Auto Regressive - Moving Average Method (ARMAX) [14].

When Transmissibility is used in OMA, FRF must be known at several points and excitation force levels determined [12], [13].

One of the major difficulties in OMA is the coexistence in one signal of structural modal responses with periodic excitations due to the operation of machines fixed on the structure.

This situation is often observed in transport technologies and large industrial structures.

Several methods are proposed for separating periodic components from structural Natural modes, Mohanty [9]. Jacobsen [11] defines an indicator using the spectral description of the signal. A linear interpolation is performed on spectral responses in order to eliminate the periodic components identified. Nonetheless, this method is not suitable when the harmonic component is close to a low-damped Natural mode.

Antoni et al. [15] propose two methods for harmonic detection. The first one is based on envelope statistics and is especially applied on pure tone components. The second one is based on correlation in conjunction with an indicator built with a tuned time delay (correlation time indicator). This method is justified by the properties of the correlation (and the PSD) of random noise and periodic signals. This second method is especially suitable for non frequency modulated harmonic components. 
Antoni [16], [17] and Vrabie [18], have defined and studied properties of Spectral Kurtosis respectively for non stationary signals and stationary signals. Both methods are based on the properties of Welch's method and compute the Spectral Kurtosis in the frequency domain.

Another definition of the spectral Kurtosis, defined by Brincker [19] and Jacobsen [11], [20], [21] is based on the classical approach of the Kurtosis in a specific bandwidth.

The present work describes a method developed in order to separate structural modal responses from periodic excitation due to rotor dynamics without assumptions regarding periodic excitation.

In the continuity of previous cited works, the objective of this paper is to study and define a robust method for detection with Spectral Kurtosis. This method is applied to harmonic components detection, especially for frequency and amplitude modulated components produced by rotor dynamics.

Theoretical definitions of the SK used in cited references are presented in section 2. The several SK definitions and methods are discussed and compared with the proposed Optimized Spectral Kurtosis method (OSK) in section 4.1. The proposed OSK and the different definitions of SK are applied on experimental results and presented in section 5.

The proposed method is different from the methods used in references [16], [17], [18] because of the definition of the Spectral Kurtosis. In these references, the Kurtosis is computed from spectral data: the Fast Fourier Transform (FFT) is first apply on temporal data, and secondly the Kurtosis is computed from the Discreet Fourier Transform results. In the proposed method, the temporal signal is first filtered in the time domain with a series of sharp Pass Band Filters and secondly the Kurtosis is computed from the filtered temporal signals for each central frequency of PBF. In order to differentiate the two methods, we have called this new method the "Optimized Spectral Kurtosis" because in this new method the spectral resolution step of the FFT process is dissociated from the equivalent bandwidth used to compute the Kurtosis. This difference allows a better efficiency in the detection process. However, the efficiency of this new method depends on several parameters as the number of samples or the filtering technique. These parameters are discussed and optimized. This is the second main interest of this paper.

\section{Statistical tools and Spectral Kurtosis}

The properties of the probability density (PD) of a discrete signal make it possible to assume the nature of the initial temporal signal.

However the same probability density could be produced by an infinite number of signals. Certain deterministic signals, such as sinusoidal ones, produce singular probability densities.

The first step of the method is based on the differences observed on the PD between (Gaussian) random signals and sinusoidal signals.

In order to highlight these differences, two descriptors were selected: Peak Factor (PF) (Eq.1) and Kurtosis.

$$
P F=\frac{\operatorname{Max}\{u[n]\}}{U_{R M S}\{u[n]\}}=\frac{\operatorname{Max}\{u[n]\}}{\sqrt{\frac{1}{N} \sum_{n=1}^{N}(u[n])^{2}}}
$$

With: $\quad u[n]$ : the temporal sequence of the discrete signal, realization of the random process $X$

$U_{R M S}$ : the Root Mean Square level of the discrete signal

$N$ : number of points of the temporal sequence

The first four statistical moments allow defining the mean $\mu$ (expectation), variance (or $\sigma$ standard deviation), Skewness and Kurtosis. These descriptors about its mean and central moments are reduced: central standardized moments are divided by the standard deviation (for orders greater than 2).

$$
\begin{aligned}
& \mu_{1}=E[X] \\
& \mu_{2}=E\left[(X-\mu)^{2}\right] \\
& \mu_{3}=E\left[\left(\frac{X-\mu}{\sigma}\right)^{3}\right] \\
& \mu_{4}=E\left[\left(\frac{X-\mu}{\sigma}\right)^{4}\right]
\end{aligned}
$$

With: $\quad \mu_{1}=\mu$ : the mean

$\sigma$ : the standard deviation: $\sigma^{2}=\mu_{2}$

Kurtosis is defined by $K=\mu_{4}$ and excess Kurtosis is defined by: $K_{N}=K-3$. 
In the three signals studied, the expected values for these descriptors are presented in table 1:

\begin{tabular}{|l|c|c|c|}
\hline E\{Estimator\}lSignals & Uniform Random [-0,5 0,5] & Normal Random $\mathcal{N}(0,1)$ & Sinusoidal $[-0,50,5]$ \\
\hline Mean: $\mu_{1}$ & 0 & 0 & 0 \\
\hline Peak Factor: $P F$ & $1 /(2 \sqrt{12})$ & - & $\sqrt{2}$ \\
\hline Variance: $\sigma^{2}=\mu_{2}$ & $1 / 12$ & 1 & $1 / 8$ \\
\hline Kurtosis: $K$ & $9 / 5$ & 3 & $3 / 2$ \\
\hline
\end{tabular}

Table 1: Expected values of statistical descriptors for three signals

Brincker [19] and Jacobsen [20], [21] define the Spectral Kurtosis with the same definition used previously for the Kurtosis based on the forth statistical moment (Eq.2d) applied in a spectral bandwidth.

For signal $u$, the Spectral Kurtosis of the frequency $f$ has been previously defined and used by Antoni [17] and Vrabie [18] in a similar way:

$$
S K_{u}(f)=\frac{C_{4 u}(f)}{S_{2 u}^{2}(f)}=\frac{S_{4 u}(f)}{S_{2 u}^{2}(f)}-2
$$

$\mathrm{C}_{\mathrm{mU}}$ and $\mathrm{S}_{\mathrm{mU}}$ are respectively the $\mathrm{m}^{\text {th }}$ order cumulant and moment of a large number $\mathrm{M}$ of realizations of signal $u$.

Vrabie [18] defines the unbiased estimation of the Spectral Kurtosis $S^{*}$ for a small number $M$ of unoverlapped blocks of u who have a $\hat{U}(f)$ DFT :

$S K_{u}^{*}(f)=\frac{M}{M-1}\left[\frac{(M+1) \sum_{i=1}^{M}\left|\hat{U}_{i}(f)\right|^{4}}{\left(\sum_{i=1}^{M}\left|\hat{U}_{i}(f)\right|^{2}\right)^{2}}-2\right]$

This estimation allows defining the efficiency of detection for a signal composed of a random process and a harmonic component.

\section{Description of the method}

The main idea is based on a spectral observation of statistical descriptors. Previous works [16], [17], [18], [19], [20], [21], have been performed in defining and using the Spectral Kurtosis. Detections are defined from numerical differences of statistical descriptors between random signals and sinusoidal signals. Also, although detections are not certain they are very likely.

\subsection{Main assumptions}

The excitations highlighting normal vibration modes are assumed to be mesokurtic or leptokurtik (positive or null excess Kurtosis).

In numerical applications, the method is still pertinent provided that excess Kurtosis does not exceed -1 . 


\subsection{Global description of the method}

If the signal is composed of a sinus and a random part, Kurtosis (Eq. 2) is assumed to be between 1.5 (sinus) and 3 (Normal Random). Under the same assumptions, the PF (Eq. 1) moves within a wider range (between $\sqrt{2}$ to 5 or more).

A numerical study of sensitivity of these descriptors highlights better detection with Kurtosis than with PF. Despite a narrower range of evolution, Kurtosis proves to be more robust and reliable which is why this study was performed with Kurtosis.

The method is composed of two main steps: firstly, the harmonic components have to be detected (Section 4) and, secondly, they have to be rejected in the OMA process for modes computation. The present work focuses on the detection. When Harmonic components are detected, the OMA process reject these frequencies in the mode identification.

The detection of harmonic components is based on combining two kinds of information: statistical and spectral. The Optimized Spectral Kurtosis method (OSK) consists in establishing a spectral description of Kurtosis.

First, the signal is filtered with a narrow Pass Band Filter (PBF) centered on the frequency studied.

Secondly, the Kurtosis of the filtered signal is computed and the numerical result defines the assumed nature of the signal (included in the spectral bandwidth).

The complete spectral description of Kurtosis is obtained by a series of PBF along the entire frequency bandwidth studied. Previous steps are described in Figure 1.

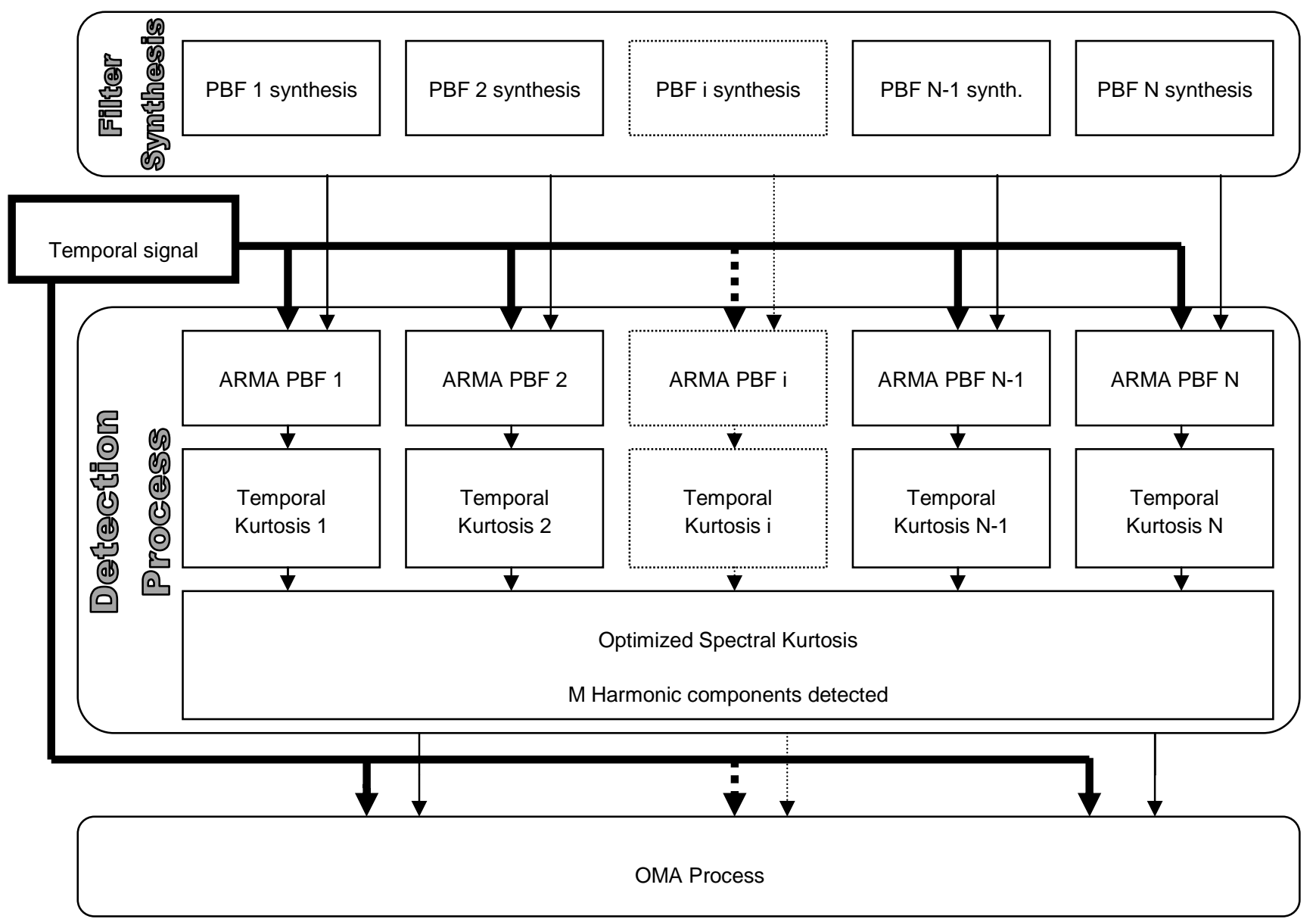

Figure 1: Block diagram of the method. Four main steps in detection : filter synthesis, signal filtering, Kurtosis computation, harmonic component detection

The optimization of the harmonic component detection with OSK had to satisfy several questions given in section 4:

1 - What is the optimal number of samples?

2 - What kind of filter should be chosen?

3 - How can the PBF be translated along the entire bandwidth?

4 - What bandwidth is best?

$5-$ What are the limits of the method? 


\section{Detailed description and critical analysis of the OSK}

First, the OSK was performed with a synthetic random signal added with a sinusoid. The random signal is a stationary Gaussian random white noise with mean $\mu=0$. The main objective of this initial approach is to highlight the efficiency and limits of application of the method in terms of optimum filter bandwidth and Signal to Noise Ratio (SNR). Results of this study are presented in figure 5.

Secondly, the OSK was performed with a synthetic random signal $r(t)$ used as an excitation source applied on a system $h(t)$ composed of two Natural modes in the frequency bandwidth studied. This system is considered as equivalent to a mechanical structure.

The output signal $s(t)$ is disturbed by a periodic signal composed of 3 harmonics. This disturbing periodic signal is considered equivalent to a rotating machine in operation and fixed on the structure (figure 2).

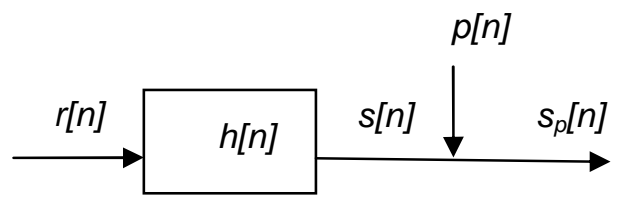

Figure 2: Block diagram of a transfer function with a polluted output

$$
s_{p}[n]=s[n]+p[n]=r[n]{ }^{*} h[n]+p[n]
$$

With: $\quad r[n]$ : Input Signal (random)

$h[n]: \quad$ Impulse Response of the system

$s[n]: \quad$ Unpolluted Output Signal

$p[n]$ : Disturbing Signal - pollution (periodic or pseudo-periodic)

$s_{p}[n]: \quad$ Polluted Output Signal $s_{p}[n]=s[n]+p[n]$

\subsection{Field of the numerical study}

The OSK was applied to a signal with a number of samples between $2^{14}$ (16 284 samples) and $2^{22}$ (4 194304 samples).

An important interest of the present work consists in distinguishing the frequency resolution of the DFT process and the frequency bandwidth in which the OSK is applied. The relation between this two frequency bandwidths is studied and optimized in order to accurate the detection process. In previous studies [17], [18] the Welch's method is used for spectral computations. In Welch's method, the two kinds of bandwidth (spectral resolution and filter bandwidth) are coupled (and equal). In that case, the Welch's Method allows a rapid computation with the FFT. In the present study signal had to be filtered in the time domain with an ARMA sequence before the Kurtosis computation. The Cauer Filter [10] (used for the ARMA filter synthesis) has been chosen in order to keep a sharp bandwidth during the optimization process of the method. The sixth order results from a compromise between numerical costs and the efficiency of the filter. However if the PSD and the OSK have the same spectral resolution (equivalent bandwidth) and when the optimal filter is chosen the Welch's Method could be performed and thus allows an important reduction of the time computation.

The PBF was applied with a constant frequency bandwidth. The OSK had already been performed with $n^{\text {th }}$ octave analysis (with frequency translation and down sampling) but, the detection obtained was less effective than with a constant spectral bandwidth. In order to reduce time computation the series of filters (with fixed bandwidth) are computed before the computation of the OSK.

The study was performed with a frequency bandwidth between $\Delta f$ and $50 \Delta f$ (with $\Delta f$ being the spectral resolution of the Fast Fourier Transform (FFT) defined by $\Delta f=1 / \tau$ and $\tau$ the duration of the signal sampled)

The signals were studied with an SNR between 1 and 50. The SNR is defined only for the frequency bandwidth containing the sinusoidal component. (For other spectral bandwidth, the SNR tends towards 0 ).

$$
S N R=\frac{A^{2}}{P S D_{\left[f_{0}-\frac{k \Delta f}{2}, f_{0}+\frac{k \Delta f}{2}\right]}(r)}
$$

With sinusoidal amplitude $A$. (In the case of pseudo periodic signals, modulations should be assumed to move slowly and in a narrow range according to the sinusoidal frequency). The Power Spectral Density of the random signal $r$ is defined for the $k \Delta f$ Bandwidth, with $\mathrm{k}$ a positive integer number.

The Transfer Function used in the numerical study is shown in figure 3. 


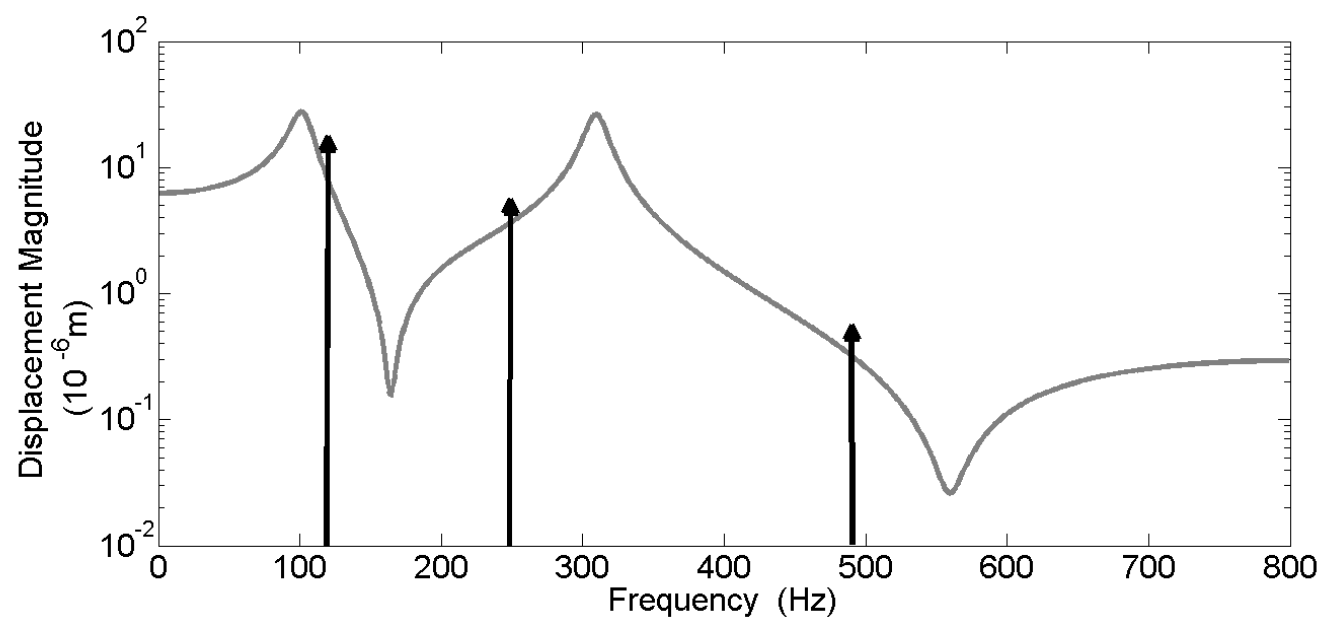

Figure 3: Test Spectral Function: a 2 DOF System excited with a random noise and a periodic signal composed with 3 frequencies

The harmonic components of the signal are $123 \mathrm{~Hz}, 246 \mathrm{~Hz}$ and $492 \mathrm{~Hz}$.

The Sampling Frequency was $1600 \mathrm{~Hz}$ and the spectral resolution $6.1 \mathrm{mHz}$ with a signal composed of $2^{18}$ samples (262 144 samples).

\subsection{OSK in operation}

Figure 4 represents the evolution of Kurtosis along the spectral domain studied. The frequency bandwidth of the filter is set at $16 \Delta f$ which is less than $0.1 \mathrm{~Hz}$. For computational reason, $\mathrm{k}$ is a $2^{\mathrm{m}}$ integer and has to be between 15 and 20 (for an optimized detection).

In this case, the detection of the three harmonic components is clearly highlighted.

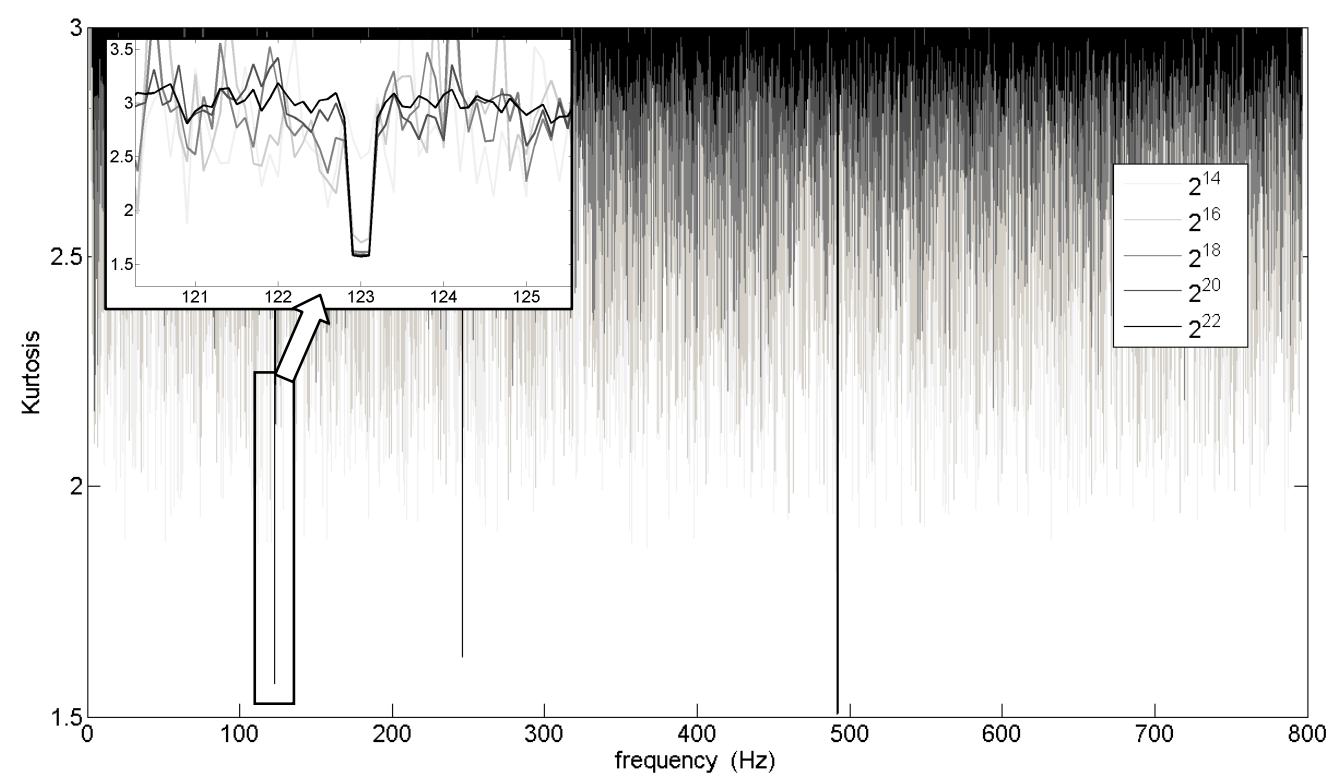

Figure 4: OSK for several numbers of samples

The method was applied to a signal described with $2^{18}$ samples for several values of SNR and several spectral bandwidths of the PBF. Detection with OSK is represented on the map in figure 5. The efficiency qualification is based on the difference (in terms of standard deviation $\sigma_{\mathrm{SK}}$ ) between the mean of the OSK $\mu_{\mathrm{SK}}$ and the measured OSK:

"Perfect" is defined for OSK $<\mu_{S K}-5 \sigma_{S K}$, "Very good", for $\mu_{S K}-3 \sigma_{S K}>O S K>\mu_{S K}-5 \sigma_{S K}$,

"Good" for $\mu_{S K}-2 \sigma_{S K}>\mathrm{OSK}>\mu_{\mathrm{SK}}-3 \sigma_{S K}$, "Intermediate" for $\mu_{\mathrm{SK}}-\sigma_{S K}>\mathrm{OSK}>\mu_{\mathrm{SK}}-2 \sigma_{S K}$,

"Bad" for $\mu_{S K}-\sigma_{S K} / 2>\mathrm{OSK}>\mu_{\mathrm{SK}}-\sigma_{S K}$, "Very bad" for $\mu_{\mathrm{SK}}-\sigma_{S K} / 4>\mathrm{OSK}>\mu_{\mathrm{SK}}-\sigma_{S K} / 2$

"Null" for OSK in the neighborhood of the mean 


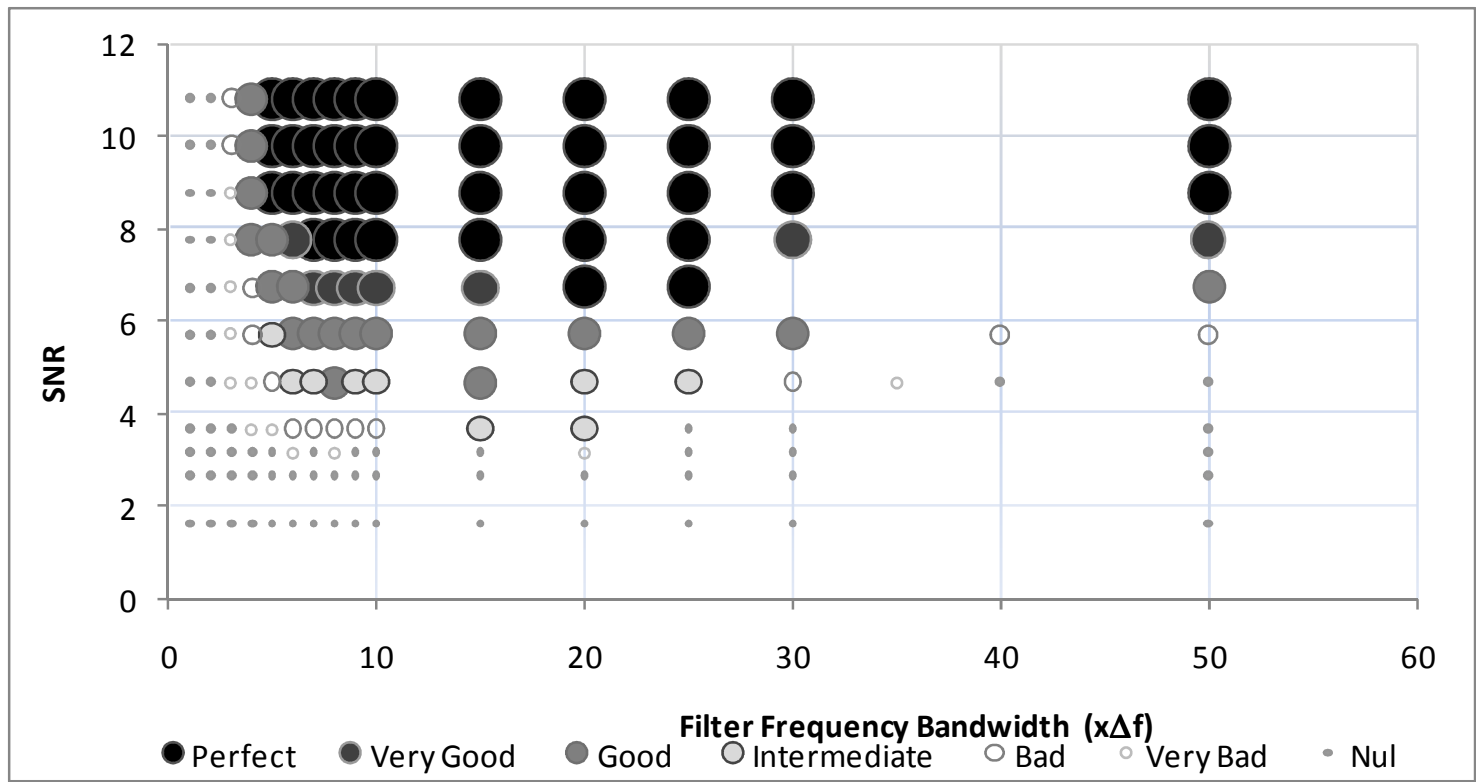

Figure 5: Efficiency of harmonic component detection for 218 samples against filter frequency bandwidth and SNR. The best OSK is obtained between 15 and 20Af bandwidth (and SNR greater than 3)

Figure 5 is built with a synthetic random signal added to a sinus and highlights:

A field in which detection is impossible for a filter bandwidth lower than or equal to $4 \Delta f$, the best detection is obtained with a filter bandwidth between 15 and $20 \Delta f$, certain detection of SNR greater than 3 and with a filter bandwidth between 15 and $20 \Delta$. 
Figure 6 shows the same kind of chart as figure 5. The limit of detection with OSK is shown for several numbers of samples.

The larger the number of samples, the better the detection. However, accurate detection could only be obtained with a large number of samples.

A reasonable compromise was found based on several numerical studies: the number of samples had to be chosen between $2^{16}$ and $2^{20}$.

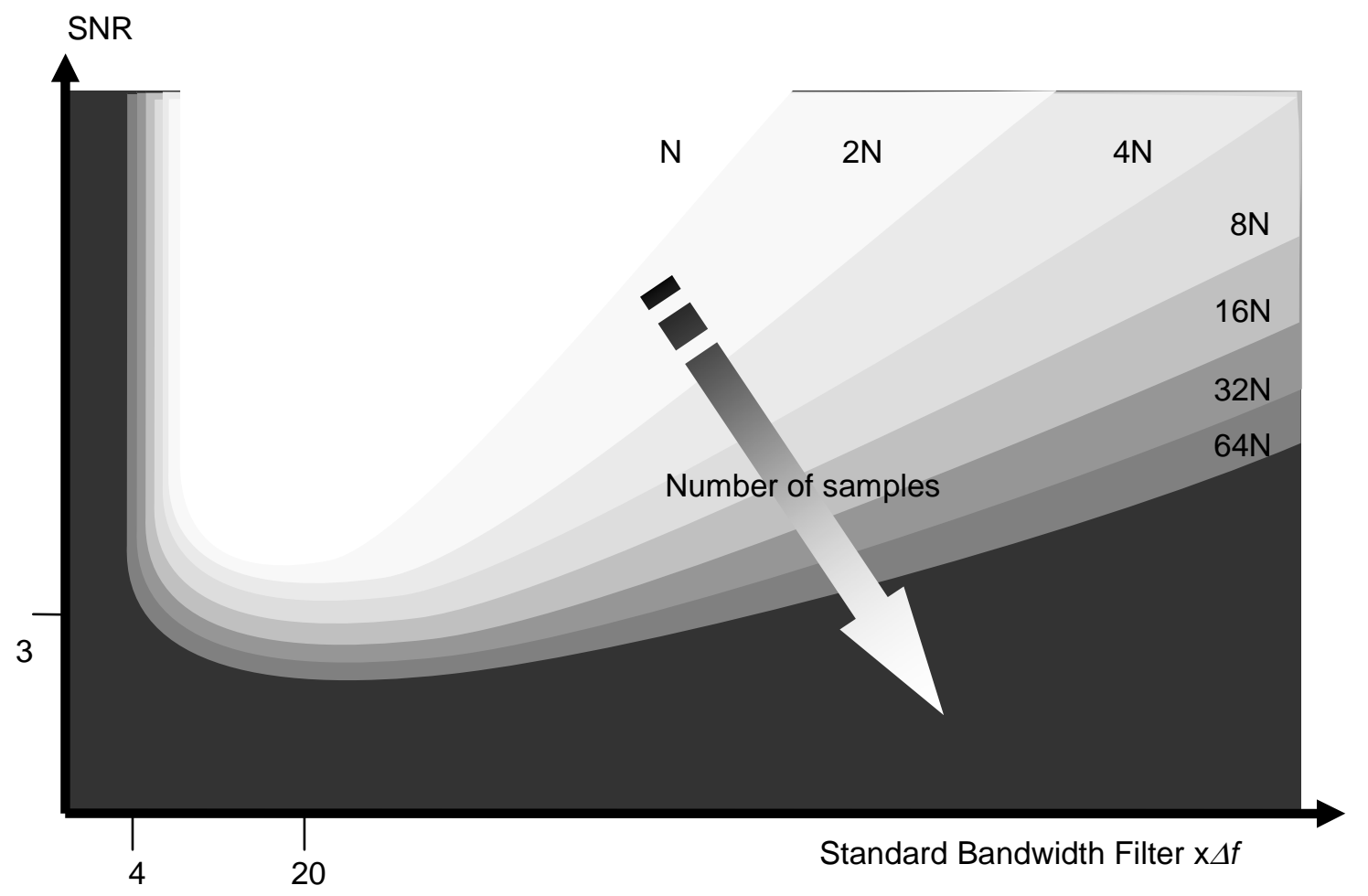

Figure 6: Chart of the detection limits for several numbers of samples White zones are obtained for very good detections

Dark zones are obtained with none detection

This chart has not been completely computed. Only few points with different number of samples have been computed in addition of figure 6. "Perfect", "Very good" and "Good" detections are considered with the same color in this chart. The more the number of samples increased, the better the detection performed.

The optimal bandwidth of the filter is close to $20 \Delta f$ and do not depends on the number of samples.

The detection could not be obtained for Bandwidth smaller than 4Af. This dark zone does not depend on the number of samples.

Over 20 $\Delta f$, the larger the bandwidth, the worse the detection. 


\section{Experimental test bench}

\subsection{Description of the experiment}

In order to test the OSK in a real experiment, a test bench was set up. The experiment is composed of two floors insulated with flexible blades (Figure 7). The mechanical system could be described as a two DOF system in the bandwidth $0-100 \mathrm{~Hz}$. The natural frequencies of the system are $9.7 \mathrm{~Hz}$ and $36.7 \mathrm{~Hz}$.

The structure is first excited with an electro-dynamic shaker and a random noise, then with an unbalanced electric engine rotating at $f b=22 \mathrm{~Hz}$ amplitude and frequency modulated in a short range. The both excitations are realized in the same time.

The aim of this experimental study is to test the efficiency of the OSK. The expected results consists in detecting the harmonic component around $f b=22 \mathrm{~Hz}$ and in not detecting them at $9.7 \mathrm{~Hz}$ and $36.7 \mathrm{~Hz}$. These two components had to be identified as natural frequencies and not as periodic excitations.

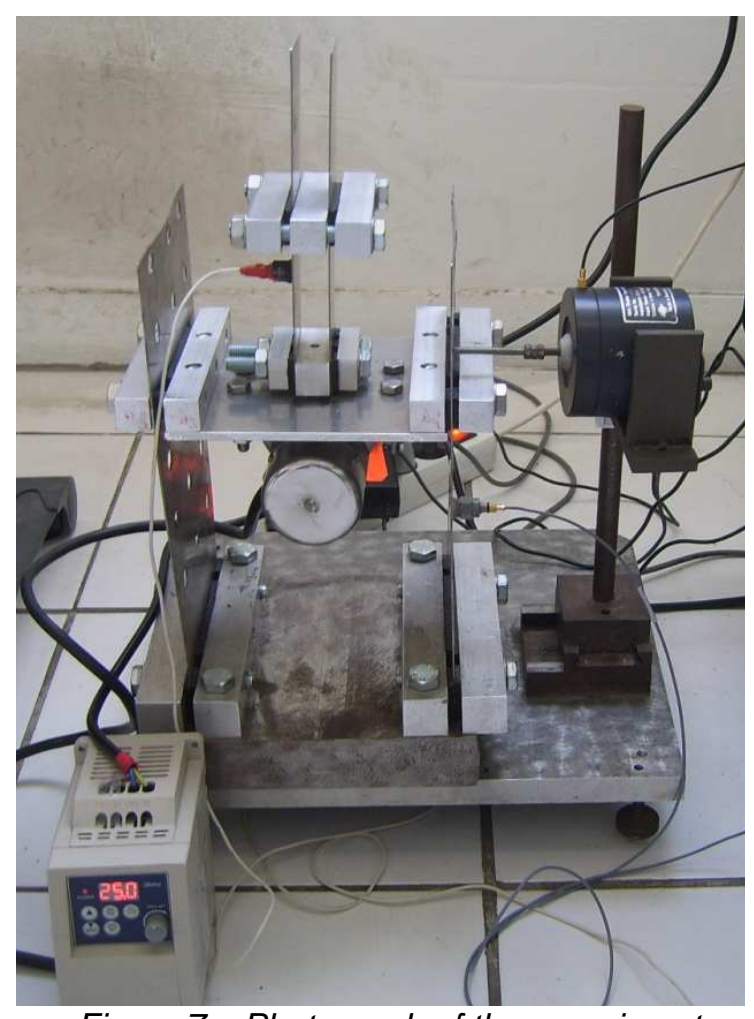

Figure 7a: Photograph of the experiments

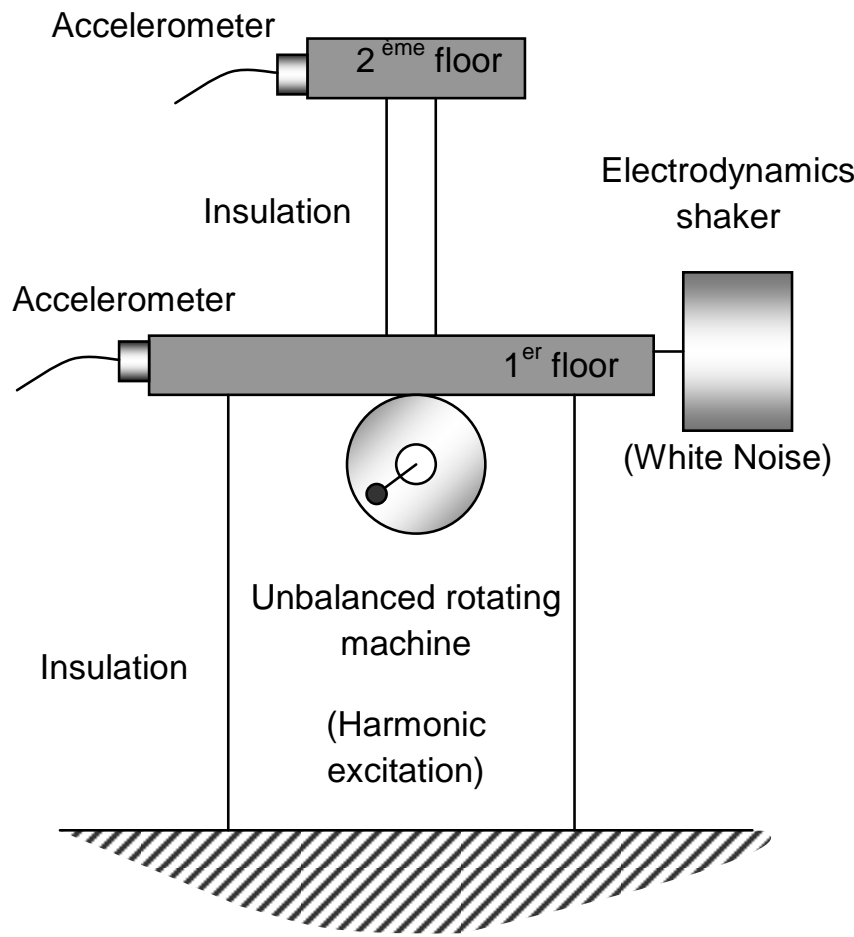

Figure 7b: Schema of the experiments 


\subsection{Experimental results}

In agreement with previous numerical studies, the method is performed with $2^{18}$ samples and Cauer filters with a bandwidth of $20 \Delta f$. For a sampling frequency of $2000 \mathrm{~Hz}$, the filter bandwidth is around $0.15 \mathrm{~Hz}$. Detection is defined for an OSK lower than 2.
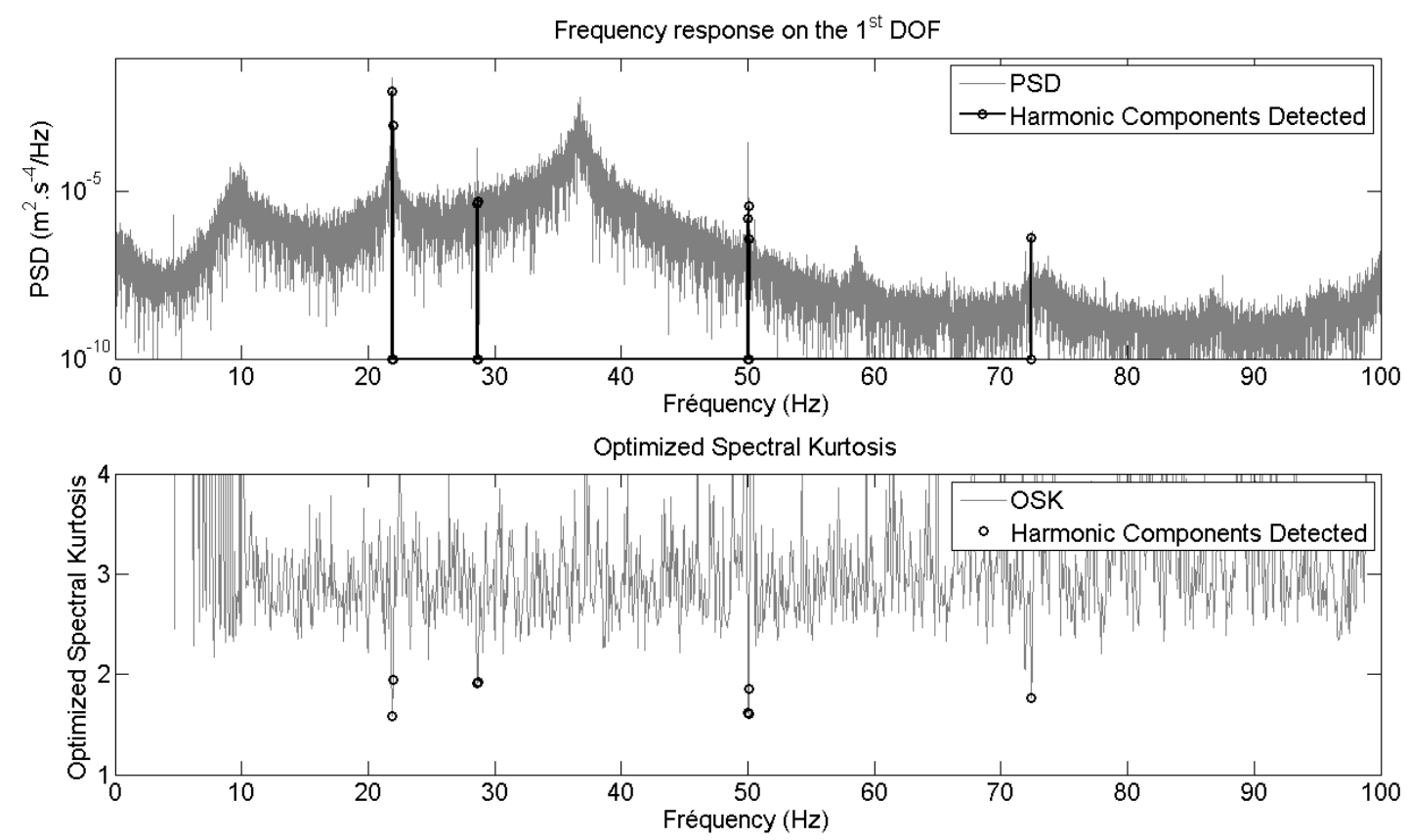

Figure 8: Detection of harmonic components using OSK 1 frequency expected but 4 frequencies detected

The results shown in figure 8 highlight the following expected observations:

Frequency $f b=22 \mathrm{~Hz}$ is well detected as a harmonic component and not as a structural response. The 2 structural Eigen modes $(9.7 \mathrm{~Hz}$ and $36.7 \mathrm{~Hz})$ are not identified as harmonic components.

Nevertheless, other components, not initially foreseen, are identified as harmonic components:

Electric network frequency $\mathrm{fr}=50 \mathrm{~Hz}$,

Two frequencies: $f r-f b=28 \mathrm{~Hz}$ and $f r+f b=72 \mathrm{~Hz}$.

These components are very small but effectively included in the signal. The first is due to the electric power frequency used for the electric engine. The two others are due to magnitude modulation between the rotation frequency of the engine and its electric power frequency.

These components are 100 to 100000 times smaller than the main excitation at $f b=22 \mathrm{~Hz}$.

Measurements have been realized with a piezoelectric accelerometer. Due to this technology, low frequencies are not studied in the present work (even if a $5 \mathrm{~Hz}$ component could be seen on figures 8 and 9 ). The unbiased Spectral Kurtosis SK* (Eq. 4) in figure 9 and the OSK figure 8 have been computed with the same record. SK* has been computed with 8, 32 and 128 unoverlapped blocs and with corresponding Hanning windows (respectively 32768, 8192 and 2048 samples). 

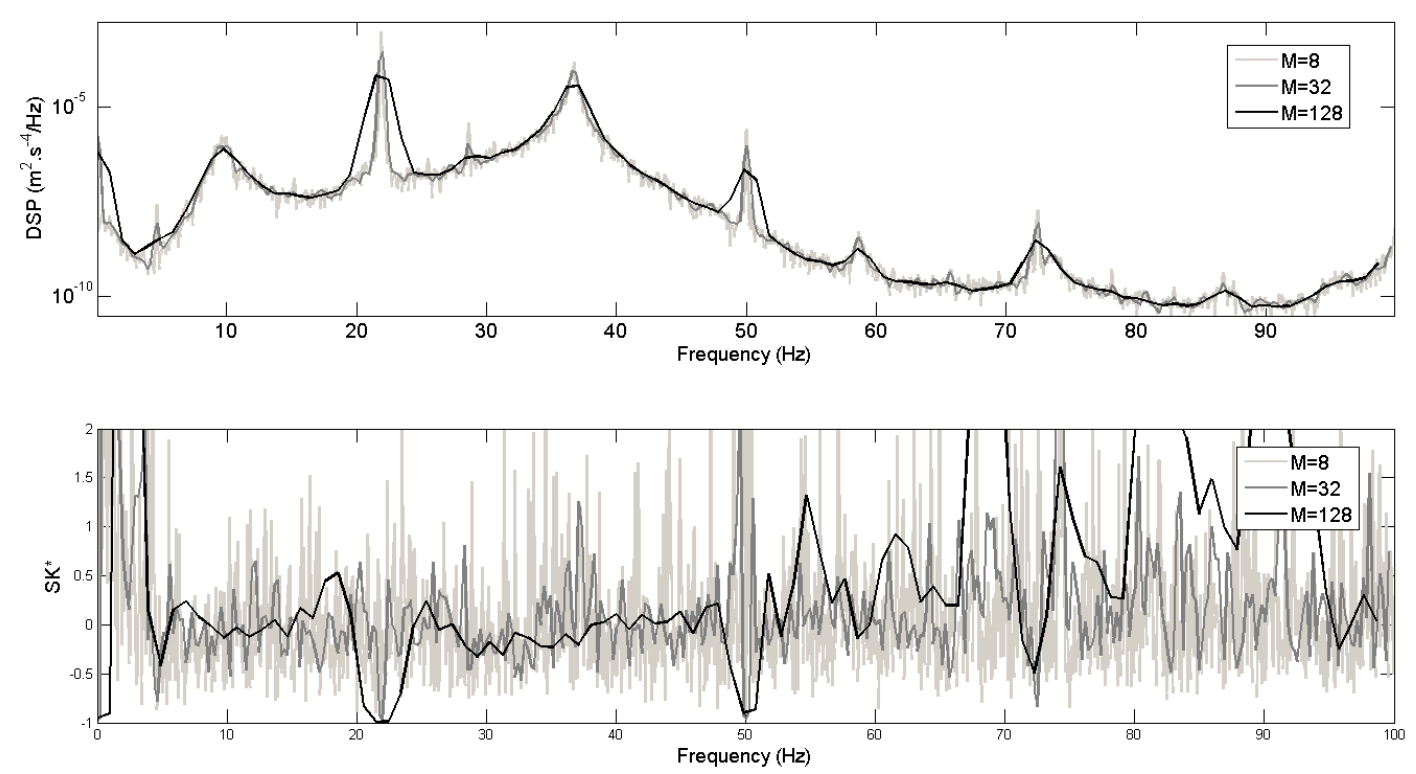

Figure 9: Detection of harmonic components with unbiased Spectral Kurtosis SK* used by Vrabie [18] and Antoni [17] for $N$ several numbers of unoverlapped blocs : due to little modulations of harmonic components the 2 main frequencies $(22 \mathrm{~Hz}$ and $50 \mathrm{~Hz})$ are hardly detected and the two others $(28 \mathrm{~Hz}$ and $72 \mathrm{~Hz})$ are $\mathrm{not}$ detected.

In this experimental study, the lack of efficiency for the $\mathrm{SK}^{*}$ is due to the use of the same bandwidth between the DFT (and PSD) and the SK. Separating both kinds of bandwidth needs to compute the SK in the time domain as it is proposed in the present paper for the OSK.

If the detection level is moved up until the $72 \mathrm{~Hz}$ component is detected, then the SK${ }^{*}$ method let appear numerous wrong detections and the choice of the number $\mathrm{N}$ of unoverlapped blocks remains a challenge.

Antoni et al.[15] propose two others detection methods for harmonic detection. The first one is based on envelope statistics and is especially applied on pure tone components. However this method could not be applied for amplitude modulated components. The second one is based on correlation in conjunction with an indicator built with a tuned time delay (correlation time indicator). This method is justified by the properties of the correlation (and the PSD) of random noise and periodic signals. However, this method is not appropriated in the case of frequency modulated signals (even with small variations of the instantaneous frequency). Due to small modulations of the 2 harmonic components $(22 \mathrm{~Hz}$ and $50 \mathrm{~Hz})$ this 2 previous methods are inappropriate for the experimental record presented in section 5.

The OSK shows that it is capable of performing beyond the initial detection target defined in this study and proves to be very efficient for harmonic component detection. 


\section{Conclusion}

Harmonic components play a role in the dynamical response of many rotating mechanical systems. However certain OMA techniques are unable to detect these kinds of signal.

The optimized OSK proposed here could detect harmonic components even when the latter were 1000 times smaller than the magnitudes observed around natural frequencies.

The OSK does not require initial knowledge of excitation forces. What is more, the natural vibration mode parameters could be obtained from only one measurement (one location) of the dynamical response.

The results of the experimental tests and comparisons with other techniques confirm the method's efficiency and the pertinence of the choice of optimum parameters in the detection process. However, as all cited methods, the OSK requires a large number of samples in order to perform the detection and its numerical computation is still costly.

\section{References}

[1] Ewins D.J., Modal Testing Theory, Practice and Application, Research Studies Press, Somerset, England, 2000.

[2] Maia S., Theoretical and Experimental Modal Analysis, Research Studies Press - England, (1997)

[3] Hermans L., Van der Auweraer H., Modal testing and analysis of structures under operational conditions: industrial applications, Mechanical System and Signal Processing 13 (2) (1999) pp. 193-216.

[4] Pintelon R., B. Peeters and P. Guillaume, Continuous-time operational modal analysis in the presence of harmonic disturbances, Mechanical Systems and Signal Processing 22 (5) (2008), pp. 1017-1035.

[5] LMS CADA-X Running Modes Manual. 1997

[6] James G.H., Carne T.G., Lauffer J.P., Sandia National Laboratories, The natural excitation technique (NExT) for modal parameter extraction from operating structures, Journal of Analytical and Experimental Modal Analysis 10 (4) (1995) pp 260-277.

[7] Brown D.L., Allemang R.J., Zimmerman R.D., Mergeay M., Parameter estimation techniques for modal analysis, SAE Transactions, SAE Paper Number 790221, Vol. 88, 1979, pp. 828-846.

[8] Ibrahim S.R., Mikulcik E.C., A method for direct identification of vibration parameters from the free response, The Shock and Vibration Bulletin 47 (4) (1977) pp. 183-198.

[9] Mohanty P., Rixen D.J., Operational modal analysis in the presence of harmonic excitations, Journal of Sound and Vibration 270 (2004) pp. 93-109.

[10] Parks, T.W., and C.S. Burrus. Digital Filter Design, New York: John Wiley \& Sons, (1987). Chapter7.

[11] Jacobsen N.J., Andersen P., Brinker R., Using enhanced frequency domain decomposition as a robust technique to harmonic excitation in operational modal analysis, in: International Conference on Noise and Vibration Engineering (ISMA), Leuven, Belgium, (September 2006).

[12] Devriendt C. and Guillaume P., The use of transmissibility measurements in output-only modal analysis, Mechanical Systems and Signal Processing 21 (7) (2007), pp. 2689-2696.

[13] Devriendt C., Steenackers G., De Sitter G. and Guillaume P. An operational modal analysis approach based on parametrically identified multivariable transmissibilities, Mechanical Systems and Signal Processing 23 (3) (2009), pp. 621-635

[14] Moore S.M., J.C.S. Lai and K. Shankar, ARMAX modal parameter identification in the presence of unmeasured excitation-I: theoretical background, Mechanical Systems and Signal Processing 21 (2007), pp. 1616-1641.

[15] Antoni J., Garibaldi L., Marchesiello S. and Sidhamed M., "New separation techniques for output-only modal analysis", Shock and Vibration, Volume 11, Numbers 3-4, Pages 227-242, 2004 
[16] Antoni J., "Blind separation of vibration components : principles and demonstrations", Mechanical Systems and Signal Processing, 19 (2005) 1166-1180

[17] Antoni J., "The spectral kurtosis: a useful tool for characterizing non-stationary signals", Mechanical Systems and Signal Processing, 20 (2006) 282-307

[18] Vrabie V.D., Granjon P., Serviere C., Spectral Kurtosis: From Definition To Application, $6^{\text {th }}$ IEEE NSPI Grado-Trieste, Italy (2003)

[19] Brincker R., Andersen P., Møller N., An Indicator for Separation of Structural and Harmonic Modes in Output-Only Modal Testing Proceedings of the 18th International Modal Analysis Conference (IMAC), San Antonio, Texas, pp.1649-1654,2000.

[20] Jacobsen N.J., Andersen P., Brincker R., Using EFDD as a Robust Technique to Deterministic Excitation in Operational Modal Analysis Proceedings of the 2nd International Operational Modal Analysis Conference (IOMAC), Copenhagen, Denmark, 2007.

[21] Jacobsen N.J., Andersen P., Brincker R., Eliminating the Influence of Harmonic Components in Operational Modal Analysis Proceedings of The 25th International Modal Analysis Conference (IMAC), Orlando, Florida, 2007. 